\title{
Multiple sexual partners and vaginal microecological disorder are associated with HPV infection and cervical carcinoma development
}

\author{
YU HUANG ${ }^{1 *}$, XINZHI WU ${ }^{1 *}$, YING LIN ${ }^{2}$, WENZHOU LI ${ }^{1}$, JIAHUA LIU $^{1}$ and BAOZHI SONG ${ }^{1}$ \\ Departments of ${ }^{1}$ Obstetrics and Gynecology and ${ }^{2}$ Pathology, \\ Shengli Clinical Medical College of Fujian Medical University and Fujian Provincial Hospital, \\ Fuzhou, Fujian 350001, P.R. China
}

Received January 20, 2020; Accepted May 27, 2020

DOI: $10.3892 / \mathrm{ol} .2020 .11738$

\begin{abstract}
There is an indirect link between multiple sexual partners (MSP) and cervical intraepithelial neoplasia (CIN) or even cervical cancer (CC). MSP may also lead to bacterial vaginosis (BV). The relationship among MSP, BV, human papillomavirus (HPV) infection and CIN/CC development in Chinese women remains unclear. The present study was designed to clarify their association. The study retrospectively analyzed 549 female patients who had visited a physical examination center. The MSP information was acquired, and vaginal microecology, HPV and cervical conization pathology (CCP) tests were performed when necessary. MSP status was distinct among patients with different levels of BV severity. In addition, as the severity of BV progressed, the HPV-positive ratio increased. Meanwhile, MSP was significantly associated with a positive HPV outcome, including HPV 16, HPV 18 and other high-risk HPV infections. The MSP group had a significantly higher percentage of positive CCP outcomes (particularly cases with CIN-II and CIN-III). Similarly, higher BV severity meant more severe CIN/CC progression. A logistic regression model based on age, MSP status and the Nugent score level was used in order to predict the CCP outcome. Furthermore, a receiver operating characteristic curve analysis resulted in an area under the curve of 0.834 . In conclusion, the combination of MSP information and BV examination may provide a rapid, economic and accurate prediction of CIN/CC. Health education on sexual behavior
\end{abstract}

Correspondence to: Dr Baozhi Song, Department of Obstetrics and Gynecology, Shengli Clinical Medical College of Fujian Medical University and Fujian Provincial Hospital, 134 East Street, Gulou, Fuzhou, Fujian 350001, P.R. China

E-mail: songbz03@163.com

*Contributed equally

Key words: bacterial vaginosis, Nugent score, cervical cancer, multiple sexual partners, human papillomavirus and timely detection/treatment of BV should be conducted to reduce the risk of $\mathrm{CC}$.

\section{Introduction}

Cervical cancer (CC) is a serious public health problem in China, with $\sim 102,000$ new cases estimated according to the National Central Cancer Registry in 2013 (1). In addition, $\mathrm{CC}$ is the second most common malignant tumor in women worldwide $(2,3)$. It is widely known that human papillomavirus (HPV) infection (4), family history of CC (5) and HIV infection (6) contribute to the development of CC. HPV infection is one of the main causes of CC. Nearly all cases of CC are causally associated with HPV infection $(7,8)$, particularly high-risk types of HPV, including HPV 16 and 18 (9-11). Simultaneously, sexual contact is the dominant risk factor for HPV infection, particularly in those with multiple sexual partners (MSP) (12). Thus, there is an indirect link between MSP and cervical intraepithelial neoplasia (CIN)/CC.

$\mathrm{CIN}$ is a precancerous lesion characterized by abnormal cell growth in the lining of the cervix. CIN can be divided into three stages, according to the degree of the lesion: CIN-I, CIN-II and CIN-III. CIN has the potential to progress to CC if left untreated; this usually takes $>10$ years (13). Moreover, having MSP has been reported as an independent risk factor of $\mathrm{CC}$ after adjusting for HPV infection $(14,15)$. Furthermore, the link between MSP and CIN/CC is considered to be partially associated with geographical region, as a meta-analysis of epidemiological studies revealed (16). Another consequence of MSP is an imbalance in the vaginal microecology, which theoretically directly leads to bacterial vaginosis (BV). A previous study reported a positive association between BV and cervical HPV infection (17). Dysregulated vaginal microbiota can enhance the risk of transient and persistent HPV infections (18), thus BV and HPV may jointly increase the risk of CIN. It was reported that BV is a cofactor of HPV and the development of CIN (19), and a significantly increased risk of CIN has been detected when BV is present $(20,21)$. However, controversial results also exist, for example, a study in 1995 reported that, in women with dyskaryotic cervical smears, the prevalence of bacterial vaginosis did not seem to be increased, 
and bacterial vaginosis did not influence the histologic changes. In addition, in women with low grade squamous intraepithelial lesion, aerobic vaginitis is very common but is not an indicator of HPV infection $(22,23)$. Since previous studies have only included a small number of samples (17-21), and the relationship among MSP, BV, HPV and CIN/CC development in Chinese women remains unclear, the present study was designed to clarify their association. Notably, the current study indicated that when information regarding MSP has been obtained, it is important to assess the severity of BV to better predict HPV and CIN/CC development in Chinese women.

\section{Materials and methods}

Patients. The present study retrospectively analyzed 549 female patients who had visited the physical examination center, outpatient and inpatient departments of Fujian Provincial Hospital (Fuzhou, China) from January 2017 to December 2019. The inclusion criteria were as follows: i) Aged between 20 and 55 years old, with regular menstruation and sexual activity history; ii) suspicion of high risk of HPV infection and BV infection; iii) 1 week before diagnosis, there was no history of sexual activity, the use of vaginal plugs, vaginal irrigation or other treatments. The exclusion criteria were: i) Menopause; ii) a history of total hysterectomy due to other diseases; iii) being pregnant; or iv) presenting with severe malignant tumors, AIDS and other immunodeficiencies.

The MSP information was acquired using a questionnaire; accordingly, recruited patients were divided into two groups: No MSP and MSP. The MSP group consisted of patients who had more than one male sexual partner in the last 6 months. The no MSP group comprised: i) Patients with a fixed male sexual partner, ii) patients who lived alone and had not had sex in the last 6 months and iii) those who lived alone but occasionally had sex with a fixed male.

Vaginal microecology test. The severity of BV was determined using a gram-stained slide and was detected with the Cro vaginal microbiological evaluation system (ShanDong Shida Si Biological Industry Co., Ltd.). The results were hierarchically represented by the Nugent Gram stain scoring system (24-27). A Nugent score of 7 to 10 was defined as severe BV, a score of 4 to 6 was defined as mild BV, and a score of 1 to 3 was defined as negative $\mathrm{BV}$.

Cobas high-risk HPV test. The fully automated Cobas x480 nucleic acid extraction instrument and Cobas z480 PCR analyzer instrument [both from Roche Diagnostics (Shanghai) Co., Ltd.] were utilized according to the manufacturer's protocol to detect HPV types 16, 18 and 12 other high-risk subtypes (types 31, 33, 35, 39, 45, 51, 52, 56, 58, 59, 66 and 68).

Cervical conization pathology (CCP). Colposcopy was ordered if a patient had abnormal thin-layer cytology test results or was highly possibly with CIN/CC development. The colposcope magnified the visual field, and acetic acid was used to swab the vaginal surface to discolor abnormal tissues and easily identify lesions. When abnormal areas were identified, colposcopy was followed by cervical conization to determine the final diagnosis $(24,25)$. Briefly, a cone-shaped tissue sample around and below the zone of the cervical squamocolumnar junction was resected. The resection included normal tissue ranging from 3 to $5 \mathrm{~mm}$ outside the abnormal tissue, and the cone depth ranged from 15 to $25 \mathrm{~mm}$. Cone cutting depth was determined by the site of the lesion. The tissue samples were then immediately sent for pathological examination. According to the CCP results, these patients were subdivided into CIN-I, CIN-II, CIN-III and CC groups. The remaining patients who had normal outcomes and did not receive $\mathrm{CCP}$ were divided into the No-Need-to-Test group.

Statistical analysis. All categorical data were expressed in frequencies and percentages; continuous data were expressed as the mean \pm standard deviation. SPSS software (version 25.0; IBM Corp.) was used for statistical analysis and data visualization. The $\chi^{2}$ test was used to compare frequencies in different groups, and one-way ANOVA was used for comparison of continuous data. The logistic regression model was established to predict the outcomes of different levels of CCP outcomes (No-need-to-test, CIN-I, CIN-II, CIN-III and CC). In addition, receiver operating characteristic (ROC) curves were generated for the prediction of CCP-positive results, and the area under the curve (AUC), as well as recommended threshold values in distinguishing different CCP outcomes, were calculated. $\mathrm{P}<0.05$ was considered to indicate a statistically significant difference.

\section{Results}

Age and MSP distribution. The demographic characteristics, including age and MSP status, are presented in Table I. A total of 549 women were included in the present study, with an average age of $36.01 \pm 8.07$ years. Of these, 309 women tested negative for $\mathrm{BV}$, and among women that tested positive for $\mathrm{BV}, 141$ were in the BV mild group and 99 were in the BV severe group, according to the Nugent score. The three groups displayed a similar age distribution with no significant differences across BV groups, whereas MSP status was distinct among patients with different levels of BV severity. For women who did not have MSP, the number in the BV-negative group was 298, whereas the number in the BV mild group was 116 , and in the BV severe group was 75 . For those with MSP, 11 women tested negative for BV, 25 women had mild BV and 24 women had severe BV. The percentage of patients with mild and severe BV were significantly increased in the MSP group $(\mathrm{P}<0.001)$.

Association between MSP, BV severity and HPV infection outcomes. Next, the present study investigated the relationship between BV severity and HPV infection. As BV severity progressed, the HPV-positive ratio increased (Table II). This association was consistent across all HPV groups, including total HPV, HPV 16, HPV 18 and other high-risk HPV infections. Furthermore, MSP was significantly associated with a HPV-positive outcome; this association was observed for HPV 16, HPV 18 and other high-risk HPV infections (Table III).

Association between MSP, BV severity and CIN/CC development. Analysis of the association between MSP and 
Table I. Age and MSP status of enrolled patients.

\begin{tabular}{|c|c|c|c|c|c|c|}
\hline Index & Total $(n=549)$ & BV negative $(n=309)$ & $B V$ mild $(n=141)$ & BV severe $(n=99)$ & $\mathrm{F}$ or $\chi^{2}$ & P-value \\
\hline Age & $36.01 \pm 8.07$ & $36.20 \pm 7.92$ & $35.44 \pm 7.92$ & $36.24 \pm 8.75$ & 0.476 & 0.622 \\
\hline MSP, n (\%) & & & & & 41.964 & $<0.001$ \\
\hline No & $489(89.1 \%)$ & $298(96.4 \%)$ & $116(82.3 \%)$ & $75(75.8 \%)$ & & \\
\hline Yes & $60(10.9)$ & $11(3.6 \%)$ & $25(17.7 \%)$ & $24(24.2 \%)$ & & \\
\hline
\end{tabular}

$\mathrm{BV}$, bacterial vaginosis; MSP, multiple sexual partners.

Table II. Association between BV severity and HPV infection.

\begin{tabular}{|c|c|c|c|c|c|}
\hline HPV infection & BV negative (\%) & BV mild (\%) & BV severe $(\%)$ & $\chi^{2}$ & $\mathrm{P}$-value \\
\hline Positive & & & & 60.833 & $<0.001$ \\
\hline No & $174(56.3)$ & 45 (31.9) & $15(15.2)$ & & \\
\hline Yes & $135(43.7)$ & $96(68.1)$ & $84(84.8)$ & & \\
\hline HPV 16 & & & & 39.000 & $<0.001$ \\
\hline No & $289(93.5)$ & $111(78.7)$ & $70(70.7)$ & & \\
\hline Yes & $20(6.5)$ & $30(21.3)$ & $29(29.3)$ & & \\
\hline HPV 18 & & & & 20.946 & $<0.001$ \\
\hline No & $298(96.4)$ & $128(90.8)$ & $82(82.8)$ & & \\
\hline Yes & $11(3.6)$ & $13(9.2)$ & $17(17.2)$ & & \\
\hline Other HPV types & & & & 41.225 & $<0.001$ \\
\hline No & $204(66.0)$ & $70(49.6)$ & $30(30.3)$ & & \\
\hline Yes & $105(34.0)$ & $71(50.4)$ & $69(69.7)$ & & \\
\hline
\end{tabular}

$\mathrm{BV}$, bacterial vaginosis; HPV, human papillomavirus.

CCP outcome demonstrated that the MSP group had a significantly higher percentage of positive CCP outcomes (Table IV). Notably, the MSP group had a higher proportion of CIN-II and CIN-III cases. Similarly, Nugent score classification was significantly associated with the CCP outcome (Table V); higher BV severity was associated with a more severe CIN/CC progression. To understand the association between MSP, BV and CCP outcome, six groups were established according to BV severity and MSP status: No-MSP-BV-negative, No-MSP-BV-mild, No-MSP-BV-severe, MSP-BV-negative, MSP-BV-mild and MSP-BV-severe. Positive CCP outcomes were significantly associated with MSP and BV; the percentage of positive CCP results was successively increased from No-MSP-BV-negative, to No-MSP-BV-mild, MSP-BV-negative, No-MSP-BV-severe, MSP-BV-mild and MSP-BV-severe (Table VI). These univariate results suggested that the combination of MSP and the Nugent score level may be helpful to predict the final CCP results.

Next, we used for the logistic two-ends regression model (Table VII) based on age (continuous variable), MSP status ( 0 , no; 1 , yes) and the Nugent score level (1, negative; 2 , mild; 3 , severe) in order to predict the CCP outcome (negative or positive). The calculated value of the logistic regression model using three variables (age, MSP and Nugent score, namely AMN) revealed an AUC of 0.834 in the ROC curve. This predictor had a sensitivity level of 0.803 and specificity level of 0.702 at the cutoff value of 0.0818 . When only two indexes (MSP and Nugent score, namely MN) were applied, an AUC of 0.789 was revealed. Meanwhile, the AUC of the single index Nugent score was 0.768 , which highlights the informative role of BV examination (Fig. 1). Together, the severity of BV infection may be used to predict CIN/CC development, with satisfactory sensitivity and accuracy, and vaginal microecology may strongly impact infection and cervical carcinogenesis.

\section{Discussion}

Sexually transmitted infections, including HPV, have been reported to be associated with the etiology of cervical carcinogenesis. Although HPV infection can be eliminated within 2 years without any complications, $1 \%$ of HPV-positive women develop CC (in the United States of America) (4). Thus, other potential risk factors need to be investigated to better predict CC. It is known that the normal vaginal microecology plays an indispensable role in preventing HPV infection and accelerating HPV clearance $(26,27)$. Abnormal vaginal microecology may be a synergistic factor of persistent HPV infection (28), which ultimately increases the risk of CIN/CC. A study with a twin cohort reported that increased vaginal microbiota diversity was closely related to HPV infection (29). HPV is a 
Table III. Association between MSP and HPV infection.

\begin{tabular}{|c|c|c|c|c|}
\hline HPV infection & No MSP $(n=489)(\%)$ & $\operatorname{MSP}(n=60)(\%)$ & $\chi^{2}$ & P-value \\
\hline Positive & & & 23.630 & $<0.001$ \\
\hline No & $226(46.2)$ & $8(13.3)$ & & \\
\hline Yes & $263(53.8)$ & $52(86.7)$ & & \\
\hline HPV 16 & & & 31.348 & $<0.001$ \\
\hline No & $433(88.5)$ & $37(61.7)$ & & \\
\hline Yes & $56(11.5)$ & $23(38.3)$ & & \\
\hline HPV 18 & & & 24.536 & $<0.001$ \\
\hline No & $462(94.5)$ & $46(76.7)$ & & \\
\hline Yes & $27(5.5)$ & $14(23.3)$ & & \\
\hline Other HPV types & & & 15.320 & $<0.001$ \\
\hline No & $285(58.3)$ & $19(31.7)$ & & \\
\hline Yes & $204(41.7)$ & $41(68.3)$ & & \\
\hline
\end{tabular}

HPV, human papillomavirus; MSP, multiple sexual partners.

Table IV. Association between MSP and CCP outcome.

\begin{tabular}{|c|c|c|c|c|}
\hline CCP outcome & No MSP $(n=489)(\%)$ & $\operatorname{MSP}(n=60)(\%)$ & $\chi^{2}$ & $\mathrm{P}$-value \\
\hline Result & & & 38.683 & $<0.001$ \\
\hline Negative & $445(91.0)$ & $38(63.3)$ & & \\
\hline Positive & $44(9.0)$ & $22(36.7)$ & & \\
\hline Level & & & 39.991 & $<0.001$ \\
\hline No-need-to-test & 443 (90.6) & $38(63.3)$ & & \\
\hline CIN-I & $2(0.4)$ & $0(0.0)$ & & \\
\hline CIN-II & $24(4.9)$ & $12(20.0)$ & & \\
\hline CIN-III & $19(3.9)$ & $10(16.7)$ & & \\
\hline $\mathrm{CC}$ & $1(0.2)$ & $0(0.0)$ & & \\
\hline
\end{tabular}

$\mathrm{CCP}$, cervical conization pathology; $\mathrm{CC}$, cervical cancer; CIN, cervical intraepithelial neoplasia; MSP, multiple sexual partners.

Table V. Association between Nugent score level and CCP outcome.

\begin{tabular}{|c|c|c|c|c|c|}
\hline CCP outcome & BV negative $(\%)$ & BV mild (\%) & BV severe $(\%)$ & $\chi^{2}$ & P-value \\
\hline Result & & & & 74.886 & $<0.001$ \\
\hline Positive & $297(96.1)$ & $123(87.2)$ & $63(63.6)$ & & \\
\hline Negative & $12(3.9)$ & $18(12.8)$ & $36(36.4)$ & & \\
\hline Level & & & & 89.104 & $<0.001$ \\
\hline No-need-to-test & $297(96.1)$ & $123(87.2)$ & $61(61.6)$ & & \\
\hline CIN-I & $0(0.0)$ & $0(0.0)$ & $2(2.0)$ & & \\
\hline CIN-II & $8(2.6)$ & $11(7.8)$ & $17(17.2)$ & & \\
\hline CIN-III & $4(1.3)$ & $7(5.0)$ & $18(18.2)$ & & \\
\hline $\mathrm{CC}$ & $0(0.0)$ & $0(0.0)$ & $1(1.0)$ & & \\
\hline
\end{tabular}

$\mathrm{CCP}$, cervical conization pathology; $\mathrm{BV}$, bacterial vaginosis; $\mathrm{CC}$, cervical cancer; $\mathrm{CIN}$, cervical intraepithelial neoplasia.

sexually transmitted infection, and increased multiple-partner sexual contact may enhance HPV transmission and CC risk (30). A collaborative study that collected data from $>15,000$ women with CIN-III/CC confirmed the relationship 
Table VI. Frequencies of positive cervical conization pathology outcome in six subgroups.

\begin{tabular}{lccc}
\hline Group & Negative $(\%)$ & Positive (\%) & $\chi^{2}$ \\
\hline No-MSP-BV-negative & $287(96.3$ & $11(3.7)$ & 95.301 \\
No-MSP-BV-mild & $106(91.4)$ & $10(8.6)$ & $<0.001$ \\
No-MSP-BV-severe & $52(69.3)$ & $23(30.7)$ & $1(9.1)$ \\
MSP-BV-negative & $10(90.9)$ & $8(32.0)$ & $13(54.2)$ \\
MSP-BV-mild & $17(68.0)$ & $11(45.8)$ &
\end{tabular}

$\mathrm{BV}$, bacterial vaginosis; MSP, multiple sexual partners.

Table VII. Variables in the logistic regression model of cervical conization pathology outcomes.

\begin{tabular}{lccccc}
\hline Variable & B & Wald & P-value & Odd ratio & 95\% CI of odd ratio \\
\hline Age & 0.095 & 22.282 & $<0.0001$ & 1.099 & $1.057-1.143$ \\
MSP or not & 1.708 & 20.551 & $<0.0001$ & 5.518 & $2.637-11.548$ \\
BV severity levels & & 44.181 & $<0.0001$ & & 2.915 \\
Mild vs. negative & 1.070 & 6.779 & 0.0090 & 11.780 & $1.303-6.523$ \\
Severe vs. negative & 2.466 & 41.582 & $<0.0001$ & $5.566-24.930$ \\
\hline
\end{tabular}

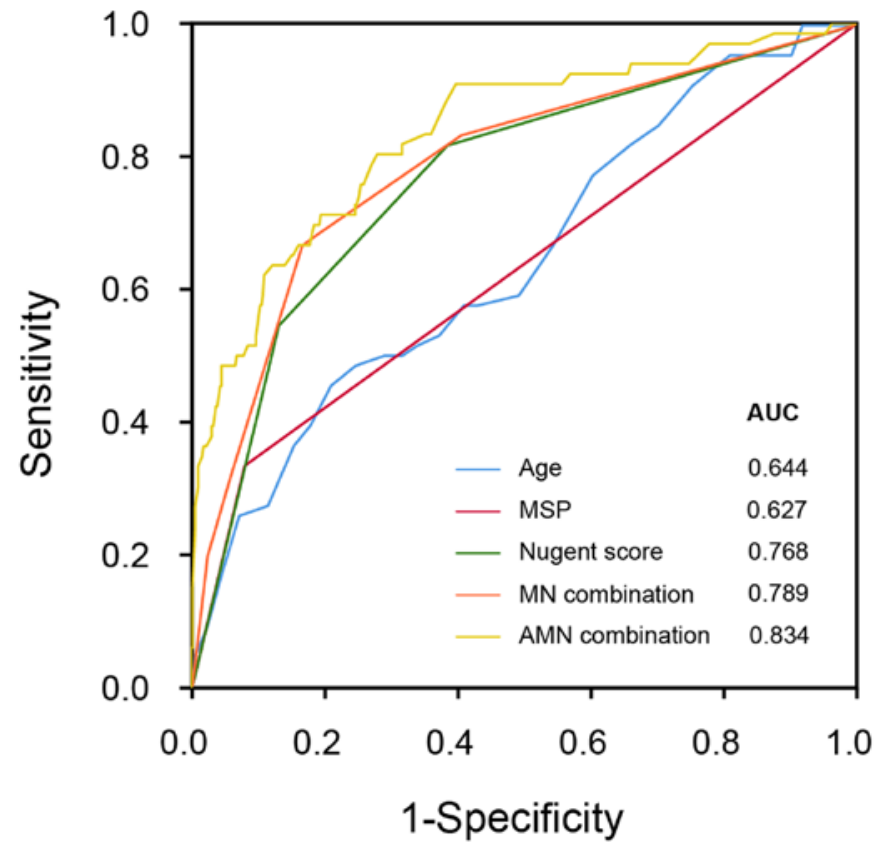

Figure 1. Receiver operating characteristic curve for efficient analysis of risk factors in CIN/CC prediction. Two variables, AMN and MN, were used to predict the outcomes of cervical conization pathology. AMN, age, MSP and Nugent score; AUC, area under the curve; MN, MSP and Nugent score; MSP: Multiple sexual partners.

between major indicators of sexual behavior and the risk of $\mathrm{CC}$ (31). Given that regional disparity can influence the relationship between MSP, BV severity, HPV and CIN/CC (16), it is worth exploring the association between these variables in Western and Eastern countries. A Spanish study reported that MSP increased the risk of HPV infection (12). In addition, a recent study revealed that marital status, age at first sexual intercourse, number of lifetime sexual partners and condom use were related to the presence of cervical pathology and the presence of oral HPV (32). Still, there have not been sufficient studies in other countries, particularly Eastern countries, investigating these associations. In the current study, a cohort of the Chinese population was used to confirm that MSP and $\mathrm{BV}$ were associated with $\mathrm{CIN} / \mathrm{CC}$ in Chinese women.

MSP has been associated with an increased risk of CIN/CC. One of the key causes of CIN/CC development is long-term infection with HPV (33). In addition, vaginal microbiota impact the acquisition, persistence and clearance of HPV, and hence affect the risk of developing CC (34). MSP status contributes to the abnormality of vaginal microecology, and this abnormal intravaginal microbial environment can be the basis for CIN/CC development. In addition, MSP may result in the introduction of other sexually transmitted pathogens, such as HIV, which is known to increase the risk of CIN/CC (35).

In the present study, patients potentially infected with HPV and $\mathrm{BV}$ were enrolled to clarify the relationships between MSP, BV and CIN/CC development. MSP and different levels of BV severity were found to be associated with HPV infection and CIN/CC. Through logistic regression analysis of CCP outcomes, MSP status and Nugent score were revealed to be risk factors for CIN/CC prediction. Using two-parameter $(\mathrm{MN})$ or three-parameter (AMN) prediction models, results obtained showed a satisfactory accuracy and sensitivity for the prediction of CIN/CC.

Overall, the findings of the current study are consistent with previously published articles. According to a study of 211 cervical samples, BV and inflammatory response were inde- 
pendently associated with the severity of cervical neoplasia in HPV-positive women (36). A meta-analysis also provided the same association between BV and uterine cervical HPV infection (17). The mechanisms underlying this association may include the production of carcinogenic nitrosamines and an altered inflammatory cytokine profile based on higher vaginal $\mathrm{pH}$ in $\mathrm{BV}$-infected patients with persistent HPV $(37,38)$. However, more mechanisms need to be explored. It has been suggested that HPV examination provides $60-70 \%$ higher protection against invasive $\mathrm{CC}$ and it is usually regarded as a more direct way of early warning of $\mathrm{CC}$ than cytological methods (39). The present work highlights the role of vaginal microecological testing in the monitoring of CIN/CC. In addition, it was confirmed that $\mathrm{BV}$ might be involved in the pathological process of CIN/CC. In the BV mild stage, the percentage of positive CCP cases sharply increased in comparison with BV negative. Therefore, even for patients with mild $\mathrm{BV}$, timely detection and treatment should be conducted to halt the development of CIN/CC.

There are some limitations in the present study. Firstly, this cross-sectional study lacks longitudinal data and the causality of the MSP-BV-HPV-CIN axis (as well as other potential causal chains) remains unclear. Secondly, the single-index MSP used in this study is not informative enough, which only concerns the total number of sexual partners. Other sexual behaviors, such as age at first intercourse and the definite number of sexual partners, were not included in this study. Moreover, the MSP index only focused on sexual activity with men, but not women. Finally, HPV 16 and HPV 18 were primarily used as the first-choice risk factors, but other high-risk HPV strains, such as HPV 31, HPV 33 and HPV 35 that are implicated in $99 \%$ of CC cases have not been analyzed $(9,40)$.

In conclusion, MSP and BV severity may be the main risk factors for HPV and CC development among Chinese women. A combination of MSP information and BV examination could provide a rapid, economic and accurate prediction of CIN/CC. Therefore, appropriate sexual health education, and prompt detection and treatment of BV could reduce the risk of CC.

\section{Acknowledgements}

Not applicable.

\section{Funding}

This work was funded by The Natural Science Foundation of Fujian Province (grant no. 2017J01254).

\section{Availability of data and materials}

The datasets used and/or analyzed during the present study are available from the corresponding author on reasonable request.

\section{Authors' contributions}

XW, YL and WL collected data. YH, XW and YL analyzed data and made the figures. JL and BS designed the study and wrote the paper. All authors read and approved the final study.

\section{Ethics approval and consent to participate}

This study was approved by the Hospital Ethics Committee of Shengli Clinical Medical College of Fujian Medical University (approval no. K2017-03-002). Written informed consent was obtained from all participants, and the consent was in strict accordance with The Declaration of Helsinki.

\section{Patients consent for publication}

Not applicable.

\section{Competing interests}

The authors declare that they have no competing interests.

\section{References}

1. Gu XY, Zheng RS, Sun KX, Zhang SW, Zeng HM, Zou XN, Chen WQ and He J: Incidence and mortality of cervical cancer in China, 2014. Zhonghua Zhong Liu Za Zhi 40: 241-246, 2018 (In Chinese).

2. Bray F, Ferlay J, Soerjomataram I, Siegel RL, Torre LA and Jemal A: Global cancer statistics 2018: GLOBOCAN estimates of incidence and mortality worldwide for 36 cancers in 185 countries. CA Cancer J Clin 68: 394-424, 2018.

3. Wardak S: Human Papillomavirus (HPV) and cervical cancer. Med Dosw Mikrobiol 68: 73-84, 2016.

4. Khan MJ, Partridge EE, Wang SS and Schiffman M: Socioeconomic status and the risk of cervical intraepithelial neoplasia grade 3 among oncogenic human papillomavirus DNA-positive women with equivocal or mildly abnormal cytology. Cancer 104: 61-70, 2005.

5. Negri E, La Vecchia C, Bosetti C, Franceschi S and Parazzini F: Risk of cervical cancer in women with a family history of breast and female genital tract neoplasms. Int J Cancer 117: 880-881, 2005.

6. Abraham AG, D'Souza G, Jing Y, Gange SJ, Sterling TR, Silverberg MJ, Saag MS, Rourke SB, Rachlis A, Napravnik S, et al: Invasive cervical cancer risk among HIV-infected women: A North American multicohort collaboration prospective study. J Acquir Immune Defic Syndr 62: 405-413, 2013.

7. Joung JG, June S and Zhang BT: Protein sequence-based risk classification for human papillomaviruses. Comput Biol Med 36: 656-667, 2006.

8. de Villiers EM, Fauquet C, Broker TR, Bernard HU and zur Hausen H: Classification of papillomaviruses. Virology 324: 17-27, 2004.

9. Boulet G, Horvath C, Vanden Broeck D, Sahebali S and Bogers J: Human papillomavirus: E6 and E7 oncogenes. Int J Biochem Cell Biol 39: 2006-2011, 2007.

10. zur Hausen H: Human papillomaviruses in the pathogenesis of anogenital cancer. Virology 184: 9-13, 1991.

11. Walboomers JM, Jacobs MV, Manos MM, Bosch FX, Kummer JA, Shah KV, Snijders PJ, Peto J, Meijer CJ and Muñoz N: Human papillomavirus is a necessary cause of invasive cervical cancer worldwide. J Pathol 189: 12-19, 1999.

12. Vaccarella S, Franceschi S, Herrero R, Muñoz N, Snijders PJ, Clifford GM, Smith JS, Lazcano-Ponce E, Sukvirach S, Shin HR, et al: Sexual behavior, condom use, and human papillomavirus: Pooled analysis of the IARC human papillomavirus prevalence surveys. Cancer Epidemiol Biomarkers Prev 15: 326-333, 2006.

13. Bos AB, van Ballegooijen M, van Oortmarssen GJ, van Marle ME, Habbema JD and Lynge E: Non-progression of cervical intraepithelial neoplasia estimated from population-screening data. $\mathrm{Br}$ J Cancer 75: 124-130, 1997.

14. Herrero R, Brinton LA, Reeves WC, Brenes MM, Tenorio F, de Britton RC, Gaitan E, Garcia M and Rawls WE: Sexual behavior, venereal diseases, hygiene practices, and invasive cervical cancer in a high-risk population. Cancer 65: 380-386, 1990.

15. Wang PD and Lin RS: Risk factors for cervical intraepithelial neoplasia in Taiwan. Gynecol Oncol 62: 10-18, 1996. 
16. Liu ZC, Liu WD, Liu YH, Ye XH and Chen SD: Multiple sexual partners as a potential independent risk factor for cervical cancer: A meta-analysis of epidemiological studies. Asian Pac J Cancer Prev 16: 3893-3900, 2015.

17. Gillet E, Meys JF, Verstraelen H, Bosire C, De Sutter P, Temmerman $\mathrm{M}$ and Broeck DV: Bacterial vaginosis is associated with uterine cervical human papillomavirus infection: A meta-analysis. BMC Infect Dis 11: 10, 2011.

18. Brotman RM, Shardell MD, Gajer P, Tracy JK, Zenilman JM, Ravel J and Gravitt PE: Interplay between the temporal dynamics of the vaginal microbiota and human papillomavirus detection. J Infect Dis 210: 1723-1733, 2014.

19. Platz-Christensen JJ, Sundstrom E and Larsson PG: Bacterial vaginosis and cervical intraepithelial neoplasia. Acta Obstet Gynecol Scand 73: 586-588, 1994

20. Platz-Christensen JJ, Larsson PG, Sundstrom E and Bondeson L: Detection of bacterial vaginosis in Papanicolaou smears. Am J Obstet Gynecol 160: 132-133, 1989.

21. Verbruggen BS, Boon ME and Boon LM: Dysbacteriosis and squamous (pre)neoplasia of immigrants and Dutch women as established in population-based cervical screening. Diagn Cytopathol 34: 377-381, 2006.

22. Peters N, Van Leeuwen AM, Pieters WJ, Hollema H, Quint WG and Burger MP: Bacterial vaginosis is not important in the etiology of cervical neoplasia: A survey on women with dyskaryotic smears. Sex Transm Dis 22: 296-302, 1995.

23. Jahic M, Mulavdic M,Hadzimehmedovic A and Jahic E: Association between aerobic vaginitis, bacterial vaginosis and squamous intraepithelial lesion of low grade. Med Arch 67: 94-96, 2013

24. Grubisić G, Klarić P, Jokanović L, Soljacić Vranes H, Grbavac I and Bolanca I: Diagnostic approach for precancerous and early invasive cancerous lesions of the uterine cervix. Coll Antropol 33: 1431-1436, 2009

25. Partridge EE, Abu-Rustum N, Campos S, Fahey PJ, Greer BE, Lele SM, Lieberman RW, Lipscomb GH, Morgan M, Nava ME, et al: Cervical cancer screening. J Natl Compr Canc Netw 6: 58-82, 2008.

26. Champer M, Wong AM, Champer J, Brito IL, Messer PW, Hou JY and Wright JD: The role of the vaginal microbiome in gynaecological cancer. BJOG 125: 309-315, 2018.

27. Liang Y, Chen M, Qin L, Wan B and Wang H: A meta-analysis of the relationship between vaginal microecology, human papillomavirus infection and cervical intraepithelial neoplasia. Infect Agent Cancer 14: 29, 2019.

28. Di Paola M, Sani C, Clemente AM, Iossa A, Perissi E, Castronovo G, Tanturli M, Rivero D, Cozzolino F, Cavalieri D, et al: Characterization of cervico-vaginal microbiota in women developing persistent high-risk human papillomavirus infection. Sci Rep 7: 10200, 2017.

29. Lee JE, Lee S, Lee H, Song YM, Lee K, Han MJ, Sung J and Ko G: Association of the vaginal microbiota with human papillomavirus infection in a Korean twin cohort. PLoS One 8: e63514, 2013.

30. International Collaboration of Epidemiological Studies of Cervical Cancer: Comparison of risk factors for invasive squamous cell carcinoma and adenocarcinoma of the cervix: Collaborative reanalysis of individual data on 8,097 women with squamous cell carcinoma and 1,374 women with adenocarcinoma from 12 epidemiological studies. Int J Cancer 120 : 885-891, 2007.
31. International Collaboration of Epidemiological Studies of Cervical Cancer: Cervical carcinoma and sexual behavior: Collaborative reanalysis of individual data on 15,461 women with cervical carcinoma and 29,164 women without cervical carcinoma from 21 epidemiological studies. Cancer Epidemiol Biomarkers Prev 18: 1060-1069, 2009.

32. Sánchez-Siles M, Remezal-Solano M, López-López AM and Camacho-Alonso F: Prevalence of human papillomavirus in the saliva of sexually active women with cervical intraepithelial neoplasias. Med Oral Patol Oral Cir Bucal 25: e195-e204, 2020.

33. Kjellberg L, Hallmans G, Ahren AM, Johansson R, Bergman F, Wadell G, Angström T and Dillner J: Smoking, diet, pregnancy and oral contraceptive use as risk factors for cervical intra-epithelial neoplasia in relation to human papillomavirus infection. Br J Cancer 82: 1332-1338, 2000.

34. Mitra A, MacIntyre DA, Marchesi JR, Lee YS, Bennett PR and Kyrgiou M: The vaginal microbiota, human papillomavirus infection and cervical intraepithelial neoplasia: What do we know and where are we going next? Microbiome 4: 58, 2016.

35. Silverberg MJ, Lau B, Justice AC, Engels E, Gill MJ, Goedert JJ, Kirk GD, D'Souza G, Bosch RJ, Brooks JT, et al: Risk of anal cancer in HIV-infected and HIV-uninfected individuals in North America. Clin Infect Dis 54: 1026-1034, 2012.

36. deCastro-SobrinhoJM,Rabelo-Santos SH,Fugueiredo-Alves RR, Derchain S, Sarian LO, Pitta DR, Campos EA and Zeferino LC: Bacterial vaginosis and inflammatory response showed association with severity of cervical neoplasia in HPV-positive women. Diagn Cytopathol 44: 80-86, 2016.

37. Pavić N: Is there a local production of nitrosamines by the vaginal microflora in anaerobic vaginosis/trichomoniasis? Med Hypotheses 15: 433-436, 1984.

38. Hudson MM, Tidy JA, McCulloch TA and Rogstad KE: When is bacterial vaginosis not bacterial vaginosis?-a case of cervical carcinoma presenting as recurrent vaginal anaerobic infection. Genitourin Med 73: 306-307, 1997.

39. Ronco G, Dillner J, Elfström KM, Tunesi S, Snijders PJ, Arbyn M, Kitchener H, Segnan N, Gilham C, Giorgi-Rossi P, et al: Efficacy of HPV-based screening for prevention of invasive cervical cancer: Follow-up of four European randomised controlled trials. Lancet 383: 524-532, 2014.

40. Bruni L, Diaz M, Castellsague X, Ferrer E, Bosch FX and de Sanjose S: Cervical human papillomavirus prevalence in 5 continents: Meta-analysis of 1 million women with normal cytological findings. J Infect Dis 202: 1789-1799, 2010.

This work is licensed under a Creative Commons Attribution-NonCommercial-NoDerivatives 4.0 International (CC BY-NC-ND 4.0) License. 